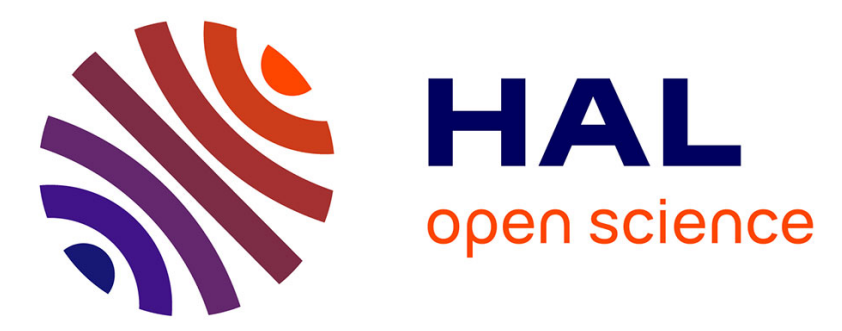

\title{
Development of a hybrid SmEdA-SEA model for predicting the power exchanged between low and high modal density subsystems
}

\author{
Guang Zhu, Laurent Maxit, Nicolas Totaro, Alain Le Bot
}

\section{To cite this version:}

Guang Zhu, Laurent Maxit, Nicolas Totaro, Alain Le Bot. Development of a hybrid SmEdA-SEA model for predicting the power exchanged between low and high modal density subsystems. Internoise, Aug 2021, Washington (virtual), United States. 10.3397/IN-2021-2535 . hal-03427208

\author{
HAL Id: hal-03427208 \\ https://hal.science/hal-03427208
}

Submitted on 13 Nov 2021

HAL is a multi-disciplinary open access archive for the deposit and dissemination of scientific research documents, whether they are published or not. The documents may come from teaching and research institutions in France or abroad, or from public or private research centers.
L'archive ouverte pluridisciplinaire $\mathbf{H A L}$, est destinée au dépôt et à la diffusion de documents scientifiques de niveau recherche, publiés ou non, émanant des établissements d'enseignement et de recherche français ou étrangers, des laboratoires publics ou privés. 


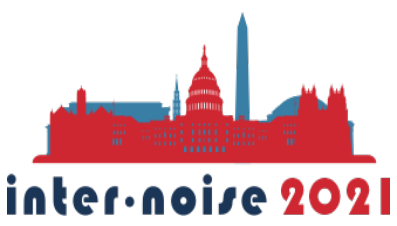

\section{Development of a hybrid SmEdA-SEA model for predicting the power exchanged between low and high modal density subsystems}

Guang $\mathrm{ZHU}^{1}$

LVA, EA677, INSA Lyon, Univ Lyon

69621 Villeurbanne, France

Laurent Maxit ${ }^{2}$

LVA, EA677, INSA Lyon, Univ Lyon

69621 Villeurbanne, France

Nicolas Totaro ${ }^{3}$

LVA, EA677, INSA Lyon, Univ Lyon

69621 Villeurbanne, France

Alain LE BOT ${ }^{4}$

LTDS, CNRS UMR 5513, École Centrale de Lyon

69134 Écully, France

\begin{abstract}
Statistical modal Energy distribution Analysis (SmEdA) was developed from classical Statistical Energy Analysis (SEA). It allows computing power flow between coupled subsystems from the deterministic modes of uncoupled subsystems without assuming the SEA modal energy equipartition. SmEdA is well adapted in mid-frequency when the subsystems have not a very high modal density. However, for some systems e.g. the plate cavity system, one subsystem can exhibit a low modal density while the other one a high one. The goal of the paper is then to propose an extension of SmEdA formulation that allows describing one subsystem by its deterministic modes, and the other one supposing modal energy equipartition and a diffuse field. The uncertain subsystem is then characterized by sets of natural frequencies and mode shapes constructed based on the Gaussian Orthogonal Ensemble matrix and the cross-spectrum density of a diffuse field, respectively. This formulation permits not only the computation of mean noise response but also the variance generated by the uncertainties and furthermore without bringing in much computation. It is demonstrated that the obtained analytical results from the proposed hybrid SmEdA/SEA are consistent with that of a Monte Carol simulation which is calculated with samples having randomness in the dimension of the cavity.
\end{abstract}

\footnotetext{
${ }^{1}$ guang.zhu@insa-lyon.fr

2laurent.maxit@insa-lyon.fr

3nicolas.totaro@insa-lyon.fr

${ }^{4}$ Alain.Le-Bot@ec-lyon.fr
} 


\section{INTRODUCTION}

The vibroacoustic analysis of complex systems plays an important role in the engineering field especially for transportation equipment such as vehicles, ships, aerospace structures, etc. In highfrequency range, the uncertainties and randomness that exist inevitably in complex systems highly affect the deterministic response [1], thus Statistical Energy Analysis (SEA), which describes the vibrational behavior of each subsystem statistically with averaged energetic variables, is more widely used [2]. In classical SEA, the system is divided into subsystems and the response is characterized by the mean energy in each of the subsystems. Strictly speaking, the "mean" output is indicating the average taken over an ensemble of systems having similar properties in general but differ in details [3]. Sometimes, SEA-like (SEAL) is also used in the engineering field for convenience, in which the "mean" output is defined as a frequency-band average taken on one particular deterministic sample [4].

Several assumptions need to be fulfilled when using the SEA, which makes its range of validity mostly in the high-frequency domain [5]. To widen its application to the mid-frequency range, Statistical modal Energy distribution Analysis (SmEdA) was derived based on Dual Modal Formulation (DMF) [6]. The modal energy equipartition assumption is removed in the SmEdA derivation, therefore, SmEdA can be adapted well to systems containing low modal density subsystems [7-9]. However, when it is applied to a complex system that contains both low and high modal density subsystems (e.g. a structure-cavity system), it may encounter computation problems because the governing equation system describing the power balance of each mode may consist of thousands of linear equations. Moreover, deterministic modes of the cavity are sometimes difficult to obtain especially when the cavity is in an irregular shape.

Under this circumstance, a Hybrid method is developed by combining the advantages of SmEdA and SEA, which allows characterizing the low modal density subsystem by its deterministic modes and high modal density subsystem by a diffuse field. The diffuse field is described by a set of natural frequencies and mode shapes constructed based on the Gaussian Orthogonal Ensemble (GOE) and the cross-spectrum density of a diffuse field [10]. The ensemble-averaged response and the corresponding variance can be obtained by employing a Monte Carlo (MC) simulation with each sample consisting of one realization of the diffuse field together with the deterministic modes of the low modal density subsystem [11].

In this paper, the governing equations of SmEdA-SEAL are formulated in section 2. Section 3 introduces a diffuse model for the studied cavity, upon which the SmEdA-SEA is developed by establishing an MC simulation. The numerical calculation proceeds in section 4, the result predicted by SmEdA-SEAL is validated with DMF and SmEdA approach, while the results obtained by SmEdA-SEA are verified with that of an uncertain model that is an MC simulation consisting of deterministic samples with a certain degree of randomness.

\section{FORMULATION OF HYBRID SMEDA-SEAL}

In this section, the governing equations when using hybrid SmEdA-SEA are derived for one complex system containing low modal density subsystem 1 and high modal density subsystem 2.

\subsection{Review of SmEdA}

The SmEdA approach was derived by Maxit and Guyader from Dual Modal Formulation [12,13], in which one of the subsystems is characterized with blocked pressure modes on the coupling area, and the other is characterized with its free displacement modes. Assuming there are $N_{1}$ resonant modes in subsystem 1 and $N_{2}$ in subsystem 2 in the studied frequency band, the interactions of the modes between two subsystems are interpreted as interacted oscillators with gyroscopic coupling without dissipation of energy (no direct coupling between two modes of the same subsystem). Assuming the 
modal interaction is weak, and the external excitation are uncorrelated white noise, the time-averaged power flow $\Pi_{p q}$ between mode $p$ of subsystem 1 and mode $q$ of subsystem 2 is proportional to the difference in the time-averaged modal energies as Equation $1[14,15]$

$$
\Pi_{p q}=\beta_{p q}\left(E_{p}-E_{q}\right) .
$$

where $E_{p}$ and $E_{q}$ are the modal energies of mode $p$ and mode $q$. Modal coupling loss factor $\beta_{p q}$ can be expressed in terms of the modal information as in Equation 2 [16],

$$
\beta_{p q}=\frac{\left(W_{p q}\right)^{2}}{M_{p} M_{q}}\left[\frac{\eta_{p} \omega_{p} \omega_{q}^{2}+\eta_{q} \omega_{q} \omega_{p}^{2}}{\left(\omega_{p}^{2}-\omega_{q}^{2}\right)^{2}+\left(\eta_{p} \omega_{p}+\eta_{q} \omega_{q}\right)\left(\eta_{p} \omega_{p} \omega_{q}^{2}+\eta_{q} \omega_{q} \omega_{p}^{2}\right)}\right]
$$

where $M_{p}, \omega_{p}, \eta_{p}$ and $M_{q}, \omega_{q}, \eta_{q}$ are respectively the modal mass, natural frequency, and modal damping loss factor of mode $p$ of subsystem 1 and mode $q$ of subsytem 2 . $W_{p q}$ is the interaction modal work between mode $p$ and $q$. Considering mode $p$ of subsystem 1 and mode $q$ of subsystem 2, the principle of energy conservation yields a linear equation system on modal energies $E_{p}$ and $E_{q}$ as

$$
\begin{array}{ll}
\Pi_{i n j}^{p}=\eta_{p} \omega_{p} E_{p}+\sum_{q=1}^{N_{2}} \beta_{p q}\left(E_{p}-E_{q}\right), & \forall p \in\left[1, N_{1}\right], \\
\Pi_{i n j}^{q}=\eta_{q} \omega_{q} E_{q}-\sum_{p=1}^{N_{1}} \beta_{p q}\left(E_{p}-E_{q}\right), & \forall q \in\left[1, N_{2}\right],
\end{array}
$$

where $\Pi_{i n j}^{p}$ and $\Pi_{i n j}^{q}$ respectively represent the inject power into $p$ th mode of subsystem 1 and $q$ th mode of subsystem 2 by external white noise excitation. $\eta_{p} \omega_{p} E_{p}$ and $\eta_{q} \omega_{q} E_{q}$ are time-averaged dissipated powers by mode $p$ and model $q$. Solving this equation system yields modal energies $E_{p}$ and $E_{q}$, The total energy of each subsystem $E_{1}$ and $E_{2}$ can be obtained by adding up all the modal energies in the studied frequency band.

\subsection{Extension of SmEdA to SEAL and hybrid SmEdA-SEAL}

In classical SEA, modal energy equipartition is assumed in all the subsystems, then one can get the modal energies of $E_{p}$ and $E_{q}$ in forms of

$$
E_{p}=\frac{E_{1}}{N_{1}}, \quad E_{q}=\frac{E_{2}}{N_{2}} .
$$

Introducing this relation into Equation 3 and adding the modal energy conservation equation for each subsystem, the energy balance equation of SEAL method can be built as

$$
\begin{aligned}
& \Pi_{i n j}^{1}=\omega_{c} \eta_{1} E_{1}+\omega_{c} \eta_{12}\left(E_{1}-\frac{N_{1}}{N_{2}} E_{2}\right), \\
& \Pi_{i n j}^{2}=\omega_{c} \eta_{2} E_{2}+\omega_{c} \eta_{12}\left(\frac{N_{1}}{N_{2}} E_{2}-E_{1}\right),
\end{aligned}
$$

where $\Pi_{i n j}^{1}$ and $\Pi_{i n j}^{2}$ respectively represent the power injected into subsystem 1 and subsystem 2 , $\omega_{c} \eta_{1} E_{1}$ and $\omega_{c} \eta_{1} E_{1}$ is the power dissipated in subsystem 1 and subsystem 2 , and $\eta_{12}$ is the SEAL coupling loss factor given by,

$$
\eta_{12}=\frac{1}{N_{1} \omega_{c}} \sum_{p=1}^{N_{1}} \sum_{q=1}^{N_{2}} \beta_{p q}
$$

When SEAL is employed, the underlying assumption implies that energy equipartition can be fulfilled in all the subsystems. However, it is not necessarily true for a complex system containing a 
low modal density subsystem. It may be for instance the case for a structure-cavity system for which the structure has a low modal density whereas the cavity has a high one. To consider the situation when one subsystem is diffuse and the other is not, the hybrid SmEdA-SEA-like (SmEdA-SEAL) is presented here. Applying the equipartition assumption only to the cavity, the energy conservation equation Equation 3 becomes

$$
\begin{aligned}
& \Pi_{i n j}^{p}=\left(\eta_{p} \omega_{p}+N_{2} \gamma_{p}\right) E_{p}-\gamma_{p} E_{2}, \quad \forall p \in\left[1, N_{1}\right], \\
& \Pi_{i n j}^{2}=-\sum_{p=1}^{N_{1}} N_{2} \gamma_{p} E_{p}+\left(\sum_{p=1}^{N_{1}} \gamma_{p}+\eta_{2} \omega_{c}\right) E_{2},
\end{aligned}
$$

where $\gamma_{p}=\frac{1}{N_{2}} \sum_{q=1}^{N_{2}} \beta_{p q}$ represents the averaged modal coupling loss between mode $p$ in subsystem 1 and all the modes in subsystem 2 .

\section{FORMULATION OF HYBRID SMEDA-SEA}

The following analysis is concerned with the Hybrid SmEdA-SEA modeling, in which an uncertain system characterized by statistical properties. For simplifying the presentation, we will suppose that the uncertain subsystem is an uncertain acoustic cavity although the proposed approach can be generalized to any type of subsystem.

\subsection{Natural frequency and mode shape realizations of the uncertain subsystem}

For defining the natural frequencies of the uncertain cavity, one supposes that they can be represented by a Gaussian Orthogonal Ensemble (GOE).

The local eigenvalue spacing distribution of the GOE is very close to the Wigner distribution as

$$
P(s)=\frac{\pi s}{2} \exp \left(-\frac{\pi s^{2}}{4}\right)
$$

where $P(s) \cdot d s$ denotes the probability of having a spacing in the interval $[s, s+d s]$. To obtain the natural frequency realizations, one vector $\mathbf{s}$ consisting of random numbers from the Wigner distribution is generated as the normalized local eigenvalue spacing,

$$
\mathbf{s}=\left[s_{1}, s_{2}, s_{3}, \ldots, s_{n}\right], \quad n=2 N_{e},
$$

where $N_{e}$ denotes the expected total number of natural frequencies in the studied frequency band that can be obtained by integrating the modal density $n(\omega)$ in that frequency band,

$$
N_{e}=\int_{\omega_{l}}^{\omega_{u}} n(\omega) d \omega, \quad \text { with } \quad n(\omega)=\frac{V \omega^{2}}{3 \pi^{2} c_{0}^{3}},
$$

in which $V$ indicates the volume of the cavity and $c_{0}$ is the speed of sound. Multiplying vector $\mathbf{s}$ with averaged nearest-neighbor eigenvalue spacing $\hat{s}$ gives the unnormalized ones,

$$
S=\mathbf{s} \cdot \hat{s}=\frac{\mathbf{s}}{n\left(\lambda_{r}\right)}=\mathbf{s} \frac{2 \omega_{r}}{n\left(\omega_{r}\right)},
$$

in which $n\left(\omega_{r}\right)$ is the averaged modal density in that frequency band. Then the $n-t h$ eigenvalue can be obtained by summing the first $n-t h$ order local spacing and the lowest eigenvalue in the frequency band of interest as

$$
\lambda_{n}=\lambda_{l}+\sum_{i=1}^{n} \mathrm{~S}_{i}, \quad \lambda_{n}<\lambda_{u} .
$$


For the mode shape realization of the uncertain cavity, firstly one mode shape vector containing the modal pressure on the coupling surface is defined as

$$
\varphi_{r}:=\left[\varphi_{r}\left(\mathrm{x}_{1}\right) \quad \varphi_{r}\left(\mathrm{x}_{2}\right) \ldots \varphi_{r}\left(\mathrm{x}_{L}\right)\right]^{T} .
$$

Supposing a standard diffuse field in the cavity, the covariance function of the modal pressure at two different positions $\mathbf{x}_{\mathbf{1}}$ and $\mathbf{x}_{\mathbf{2}}$ should respect [17]:

$$
\mathbf{C}_{\mathrm{r}}:=\operatorname{Cov}\left[\varphi_{r}\left(\mathbf{x}_{\mathbf{1}}\right) \varphi_{r}\left(\mathbf{x}_{\mathbf{2}}\right)\right]=\mathrm{E}\left[\varphi_{r}\left(\mathbf{x}_{\mathbf{1}}\right) \varphi_{r}\left(\mathbf{x}_{\mathbf{2}}\right)\right]=S_{\varphi_{r} \varphi_{r}} \frac{\sin \left(\mathrm{k}_{\mathrm{r}}\left|\mathbf{x}_{\mathbf{2}}-\mathbf{x}_{\mathbf{1}}\right|\right)}{k_{r}\left|\mathbf{x}_{\mathbf{2}}-\mathbf{x}_{\mathbf{1}}\right|}
$$

where $k_{r}=\frac{\omega_{r}}{c_{0}}$ is the wavenumber corresponding to the frequency $\omega_{r} . S_{\varphi_{r} \varphi_{r}}$ is the auto-spectrum density of boundary pressure $\varphi_{r}$. The normalization condition for room pressure $p_{r}$ of a diffuse acoustic cavity with volume $V$ is

$$
\frac{1}{V} \int_{V} p_{r}^{2}(\mathrm{x}) d \mathrm{x}=\frac{\rho_{0} c_{0}^{2}}{V}
$$

Considering that a ratio of 2 between the auto-spectrum density of boundary pressure $S_{\varphi_{r} \varphi_{r}}$ and the average of the quadratic pressure mode [18],

$$
S_{\varphi_{r} \varphi_{r}}=\frac{2 \rho_{0} c_{0}^{2}}{V}
$$

Realizations of the modal pressure $\varphi_{r}$ can be achieved through an eigenvalue decomposition of the covariance matrix,

$$
\mathbf{C}_{r}:=\mathbf{A} \boldsymbol{\Sigma} \mathbf{A}^{T}
$$

The corresponding set of modal pressure on the coupling surface can be expressed as

$$
\varphi_{k, r}:=\mathbf{A} \Sigma^{\frac{1}{2}} \boldsymbol{\xi}
$$

where $\xi$ is a vector of independent, standard normal random variables that can be realized with a Gaussian random number generator.

\subsection{Hybrid SmEdA-SEA method}

The SmEdA-SEA is established based on Monte Carlo simulation as shown in the flow chart Figure 1. For example for one rectangular plate cavity coupling system, the deterministic modes of the plate (subsystem 1) can be calculated analytically for simply supported plate or by FEM for a more complex case. For the cavity (subsystem 2), realizations of its natural frequencies and mode shapes in each frequency band can be done with the process described previously. The process of modal information realization is repeated $N_{s}$ times to generate a set of different realization, which together with the deterministic modes of the plate can be regarded as the samples of the following Monte Carlo Simulation. The frequency average response of each sample is calculated with SmEdASEL, then the ensemble average response taken on all the samples is calculated in the Monte Carlo simulation. This whole process is defined as the SmEdA-SEA model.

\section{NUMERICAL RESULTS}

In this section, numerical results for one plate cavity system (Figure 2) are calculated and compared to the reference results in order to validate and assess the foregoing developed methods. 


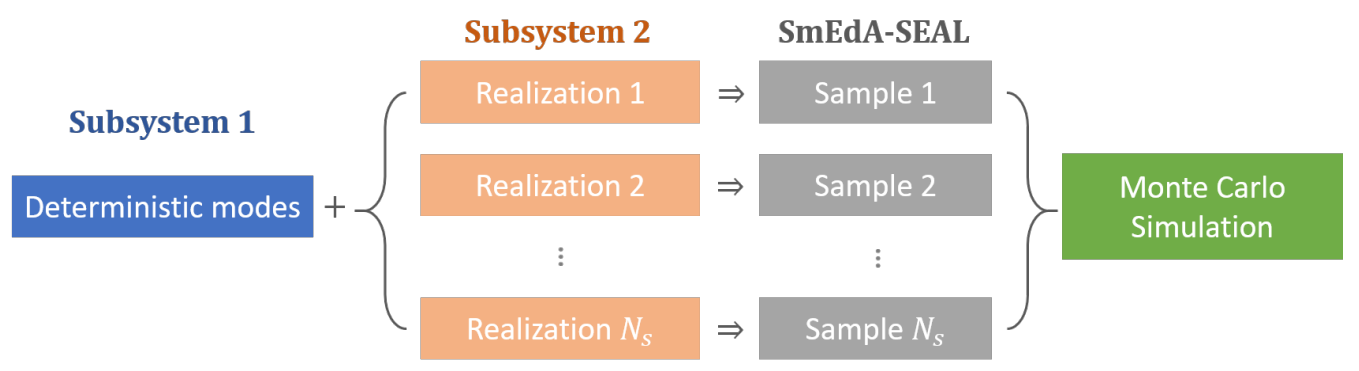

Figure 1: flow chart of SmEdA-SEA

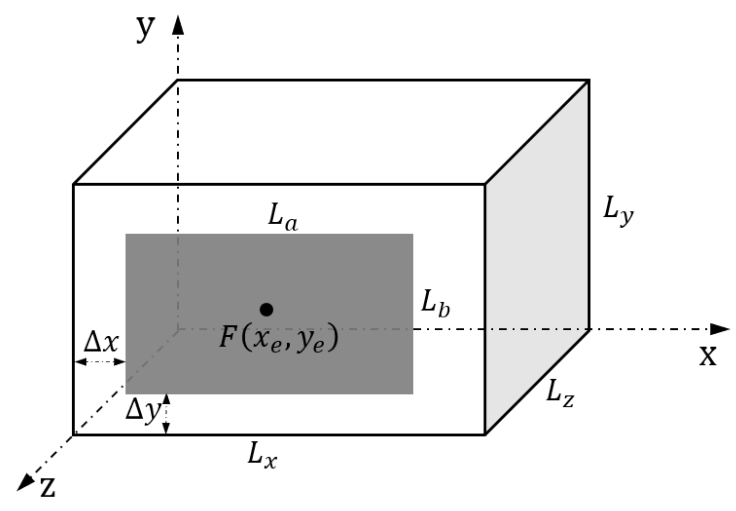

Figure 2: Simply supported plate excited by random force radiates into a cavity

\subsection{Validation of SmEdA-SEAL}

In this case, the plate, of dimension $L_{a}=0.93 \mathrm{~m}, L_{b}=0.86 \mathrm{~m}$ and thickness $h=0.001 \mathrm{~m}$ is simply supported and excited by a random force $F$ of white-noise type at position of $(0.72,0.81)$. It radiates energy into a cavity with rigid walls and the cavity is of dimension $L_{x}=1 \mathrm{~m}, L_{y}=0.9 \mathrm{~m}$, $L_{z}=0.8 \mathrm{~m}$. The position of the plate $\Delta x=0.047 \mathrm{~m}, \Delta y=0.022 \mathrm{~m}$. The Young's modulus of the plate $E=2.1 \times 10^{11} \mathrm{~Pa}$, Poisson ratio $v=0.31$ and density $\rho=7800 \mathrm{~kg} / \mathrm{m}^{3}$. The air in the cavity is of density $\rho_{0}=1.29 \mathrm{~kg} / \mathrm{m}^{3}$ and air speed $c_{0}=340 \mathrm{~m} / \mathrm{s}$. Damping coefficient for two subsystems $\eta_{1}=0.01, \eta_{2}=0.001$.

Employing the DMF, SmEdA, SEAL, and hybrid SmEdA-SEAL formulation for the plate cavity system, the averaged exchanged power between two subsystems are illustrated in Figure 3 . The results predicted by SmEdA and the hybrid SmEdA-SEAL formulation both converge well to the DMF method with a difference less than $1 \mathrm{~dB}$ from $700 \mathrm{~Hz}$. By contrast, the result predicted by the SEAL is not accurate, and the discrepancies are larger than $1 \mathrm{~dB}$ in most of the studied frequency bands. The validate range of Hybrid SmEdA-SEAL is as wide as that of the SmEdA. Furthermore, considering the mode number in the cavity can be numerous, which leads to long-time calculation and large computation resources necessary for SmEdA analysis, the hybrid formulation can be a great alternative method for this kind of complex system that contains both high and low modal density subsystems.

\subsection{Validation of SmEdA-SEA}

In this case, the plate, of dimension $0.34 \mathrm{~m} \times 0.46 \mathrm{~m} \times 0.001 \mathrm{~m}$, is excited at position of $\left(x_{e}, y_{e}\right)=$ $(0.22 \mathrm{~m}, 0.11 \mathrm{~m})$. In the numerical calculation using SmEdA-SEA, the dimension of the coupled cavity is of dimension $1.0 \mathrm{~m} \times 0.9 \mathrm{~m} \times 0.8 \mathrm{~m}$ and the relative position of the plate $\Delta x=0.55 \mathrm{~m}, \Delta y=0.32 \mathrm{~m}$. For the uncertainty model, the uncertainty is introduced into the cavity by dimension randomness in three directions meanwhile the volume of the cavity is fixed at $1.0 \mathrm{~m} \times 0.9 \mathrm{~m} \times 0.8 \mathrm{~m}$. When the vibrational field of a cavity is modeled as a diffuse field as in the SmEdA-SEA model, we suppose 


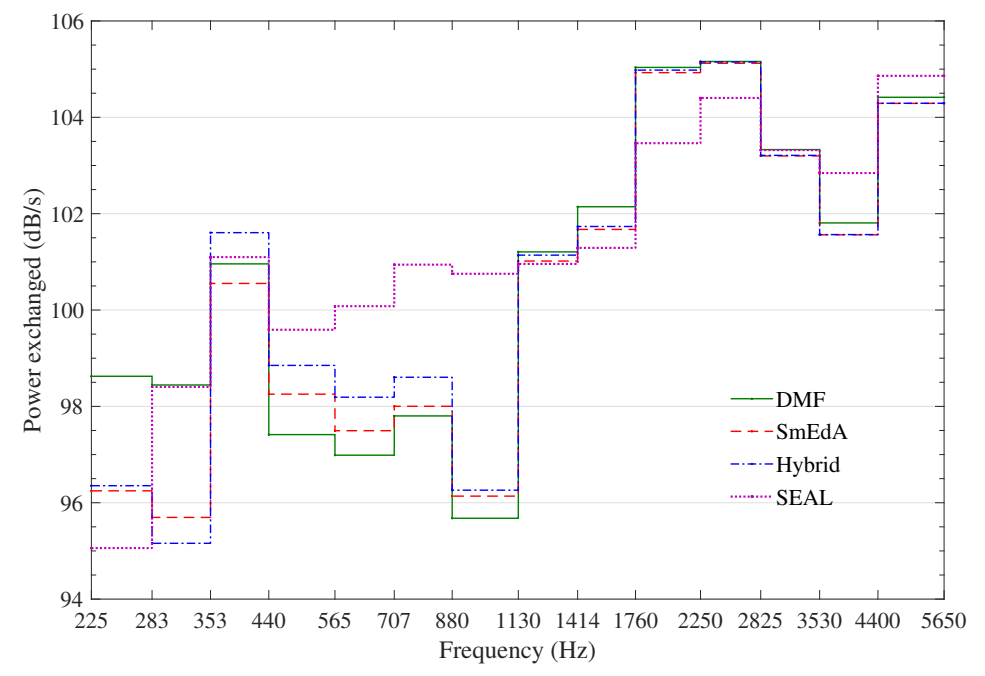

Figure 3: Exchanged power predicated by DMF, SmEdA, SEAL, and hybrid SmEdA-SEAL

that the highest degree of uncertainty is introduced into the model. Therefore theoretically speaking, the averaged exchanged power predicated by these two models should have an agreement and the confidence interval of the uncertainty model should be contained in that of the hybrid model.

Figure 4 illustrates the confidence interval and ensemble average of exchanged power predicated hybrid SmEdA-SEA compared with that of the uncertainty model with $10 \%$ uncertainty in dimension. The difference between these two models in terms of ensemble-averaged exchanged power converges to less than $1 \mathrm{~dB}$ above $880 \mathrm{~Hz}$. However, the confidence interval of the uncertainty model is not completely contained in that of the hybrid SmEdA-SEA model. The modes that participate in the response calculation of the uncertainty model are deterministic, in which some modes are sensitive to dimension uncertainty while some others are less sensitive. Therefore, the output of the uncertainty model deviates slightly from the SmEdA-SEA model. If the cavity used for the uncertainty model

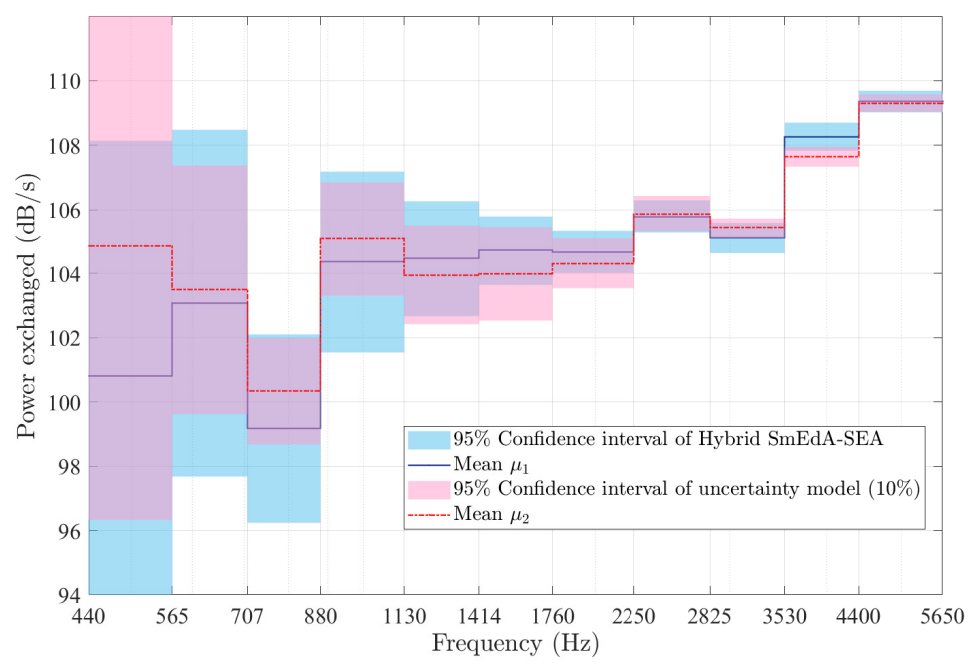

Figure 4: Confidence intervals and ensemble averages of exchanged power predicated by uncertainty model (with $10 \%$ uncertainty) and SmEdA-SEA taken on 100 samples

is ergodic, the generated vibration field becomes closer to a diffuse field, and the obtained reference result would be closer to the result of the proposed hybrid SmEdA-SEA. This is what we want to study in the next stages of this research. 


\section{CONCLUSIONS}

A hybrid SmEdA-SEA is extended from SmEdA for the vibroacoustic analysis of complex systems containing both high and low modal density subsystems. The low modal density is characterized by its deterministic modes with the SmEdA while the high modal density subsystem is assumed to be uncertain and characterized by statistical properties: GOE for the modal frequencies and diffuse field for the mode shapes. The comparison of exchanged power between two subsystems calculated by DMF, SmEdA, SEA, and SmEdA-SEAL shows a good assessment for the proposed hybrid SmEdASEAL method. In order to validate the SmEdA-SEA, the results in terms of the confidence interval and ensemble average of the exchanged power obtained by SmEdA-SEA have been compared with those obtained by an uncertainty model with random dimension. As expected, the mean level of exchanged power shows a good agreement with the uncertainty model, while their confidence interval shows a slight difference owing to the non-strict diffuse acoustic field in the uncertainty simulation. In conclusion, the present hybrid approaches are well adapted to complex systems containing subsystems with high and low modal densities. It takes fewer computation resources without diminishing the accuracy, and hybrid SmEdA-SEA allows evaluating the variance of the response generated by the uncertainty in the system without a detailed description of its underlying random parameters.

\section{ACKNOWLEDGEMENTS}

The authors are grateful for the financial support from “Institut Carnot Ingénierie@Lyon”. This work was also supported by the LABEX CeLyA (ANR-10-LABX-0060) of Université de Lyon, within the program «Investissements d'Avenir » (ANR-16-IDEX-0005) operated by the French National Research Agency (ANR).

\section{REFERENCES}

[1] Matthew Wright and Richard Weaver. New Directions in Linear Acoustics and Vibration: Quantum Chaos, Random Matrix Theory and Complexity. Cambridge University Press, New York, 2010.

[2] R.H. Lyon and R.G. Dejong. Theory and Application of Statistical Energy Analysis. Butterworth, London, 1995.

[3] Robin S. Langley. Response variance prediction in the statistical energy analysis of built-up systems. The Journal of the Acoustical Society of America, 115(2):706-718, 2004.

[4] S. Finnveden. Ensemble averaged vibration energy flows in a three-element structure. Journal of Sound and Vibration, 187(3):495-529, 1995.

[5] T. Lafont, N. Totaro, and A. Le Bot. Review of statistical energy analysis hypotheses in vibroacoustics. Proceedings of the Royal Society A: Mathematical, Physical and Engineering Sciences, 470(2162):20130515, 2014.

[6] Laurent Maxit and Oriol Guasch. A dual modal formulation for multiple flexural subsystems connected at a junction in energy-based models. Mechanical Systems and Signal Processing, 119:457-470, 2019.

[7] H.D. Hwang, L. Maxit, K. Ege, Y. Gerges, and J.-L. Guyader. Smeda vibro-acoustic modelling in the mid-frequency range including the effect of dissipative treatments. Journal of Sound and Vibration, 393:187-215, 2017.

[8] Kendra L Van Buren, Morvan Ouisse, Scott Cogan, Emeline Sadoulet-Reboul, and Laurent Maxit. Effect of model-form definition on uncertainty quantification in coupled models of mid-frequency range simulations. Mechanical Systems and Signal Processing, 93:351 - 367, September 2017. 
[9] Jie Deng, Oriol Guasch, Laurent Maxit, and Ling Zheng. Transmission loss of plates with multiple embedded acoustic black holes using statistical modal energy distribution analysis. Mechanical Systems and Signal Processing, 150:107262, 2021.

[10] P.J. Shorter and R.S. Langley. On the reciprocity relationship between direct field radiation and diffuse reverberant loading. The Journal of the Acoustical Society of America, 117(1):85-95, 2005.

[11] Cédric Van hoorickx and Edwin P.B. Reynders. Gaussian orthogonal ensemble modeling of built-up systems containing general diffuse components and parametric uncertainty. Journal of Sound and Vibration, 501:116045, 2021.

[12] L. Maxit and J.-L. Guyader. Extension of sea model to subsystems with non-uniform modal energy distribution. Journal of Sound and Vibration, 265(2):337-358, 2003.

[13] Laurent Maxit. Analysis of the modal energy distribution of an excited vibrating panel coupled with a heavy fluid cavity by a dual modal formulation. Journal of Sound and Vibration, 332(25):6703-6724, 2013.

[14] L. MAXIT and J.-L. GUYADER. Estimation of sea coupling loss factors using a dual formulation and fem modal information, part i: Theory. Journal of Sound and Vibration, 239(5):907-930, 2001.

[15] L. MAXIT and J.-L. GUYADER. Estimation of sea coupling loss factors using a dual formulation and fem modal information, part ii: Numerical applications. Journal of Sound and Vibration, 239(5):931-948, 2001.

[16] N. Totaro, C. Dodard, and J. L. Guyader. SEA Coupling Loss Factors of Complex VibroAcoustic Systems. Journal of Vibration and Acoustics, 131(4), 062009.

[17] Edwin Reynders. Generalized reverberant acoustic field modeling based on the gaussian orthogonal ensemble. In Proceedings of ISMA 2014 International Conference on Noise and Vibration Engineering, pages 2341-2356. KATHOLIEKE UNIV LEUVEN, DEPT WERKTUIGKUNDE, 2014.

[18] Jean Daniel Chazot and Jean Louis Guyader. Diffuseness and sound field distribution at room boundaries. The Journal of the Acoustical Society of America, 123(5):3504-3504, 2008. 